\title{
Materials and Devices for Organic Electronics
}

\author{
Marisol Reyes-Reyes, ${ }^{1}$ David L. Carroll, ${ }^{2}$ Werner Blau, ${ }^{3}$ and Román López-Sandoval ${ }^{4}$ \\ ${ }^{1}$ Instituto de Investigación en Comunicación Optica, Universidad Autónoma de San Luis Potosí, Alvaro Obregon 64, \\ 78000 San Luis Potosí, SLP, Mexico \\ ${ }^{2}$ Department of Physics, The Center for Nanotechnology and Molecular Materials, Wake Forest University, \\ Winston-Salem, NC 27109, USA \\ ${ }^{3}$ School of Physics, Trinity College Dublin, Dublin 2, Ireland \\ ${ }^{4}$ Division de Materiales Avanzados, IPICYT, Camino a la Presa San José 2055, Col. Lomas 4a. sección, \\ 78216 San Luis Potosí, SLP, Mexico
}

Correspondence should be addressed to Marisol Reyes-Reyes, reyesm@iico.uaslp.mx

Received 28 August 2011; Accepted 28 August 2011

Copyright (C) 2011 Marisol Reyes-Reyes et al. This is an open access article distributed under the Creative Commons Attribution License, which permits unrestricted use, distribution, and reproduction in any medium, provided the original work is properly cited.

Organic electronics have attracted much attention in recent years due to their multiple advantages such as high flexibility, easy processing, low fabrication cost, and large area fabrication. These unique advantages make them highly promising for organic solar cells, organic memory devices, organic thin film transistors, organic light emitting diodes, organic photodetectors, organic sensor, and so forth. The performance of organic electronic devices is strongly dependent on the fabrication procedure as well as the processing parameters. Among various fabrication methods to implement these organic electronics devices, spray coating is emphasized because of its compatibility to large area fabrications and industrial mass productions. However, other methods, such as inkjet printing, offer, for example, to reduce the ink material consumption by drop-on-demand design. The recent advances in fabricating organic electronic devices have been carried out with the development of novel materials. Certainly, blending of organic materials to form composites has been developed to improve the properties of the devices, or to lead studies to determine some properties due to their interaction. Complementary modeling studies can be carried out to support the evidence experimentally. In this special issue, the papers address these issues.

The first paper of this special issue is on the syntheses and characterization of new low bandgap copolymers, the results indicate that one of them has the potential for obtaining highly efficient solar cells. The second paper describes the formation and characterization of an organic molecular wire containing polypyrrolyl conjugate bonds. In addition, it is suggested that the conduction mechanism of this molecular wire might be different from semiconductors and metals. The third paper proposes, from the obtained results, that copolymer ferroelectric nanocrystals are good candidates for devices with high-density nonvolatile storage. The two subsequent papers address the influence of the processing parameters on the performance of organic memories and organic thin-film transistors. Particularly, the fourth paper presents the effect of the thickness variation of the PMMA and PSS film on the memory behavior; the different types of behavior are attributed to the metal electrodes and film thickness, whereas the organic film has minor influence. The fifth paper is on the effect of processing on film morphology and the mobility of carriers in spray-deposited organic thin film transistors. The morphology of the spray-deposited devices showing the best performance is similar with that of spin-coated devices and, for this reason, comparable performance is achieved. The sixth paper addresses the blending of materials for organic thin film transistors by using inkjet printing. Particularly, poly(3-hexylthiophene) blended with carbon nanoparticles is used as the organic semiconductor material for improving the performance of fabricated transistor. The final paper of this special issue describes the linear and nonlinear optical characterization of a composite of porphyrin with nanotube moieties. 
The results show evidence that much of the optical evidence attributed to strong noncovalent binding in these systems by other authors is likely to be an artifact. Complementary molecular dynamic studies were in striking agreement with the experimental observations.

$$
\begin{array}{r}
\text { Marisol Reyes-Reyes } \\
\text { David L. Carroll } \\
\text { Werner Blau } \\
\text { Román López-Sandoval }
\end{array}
$$



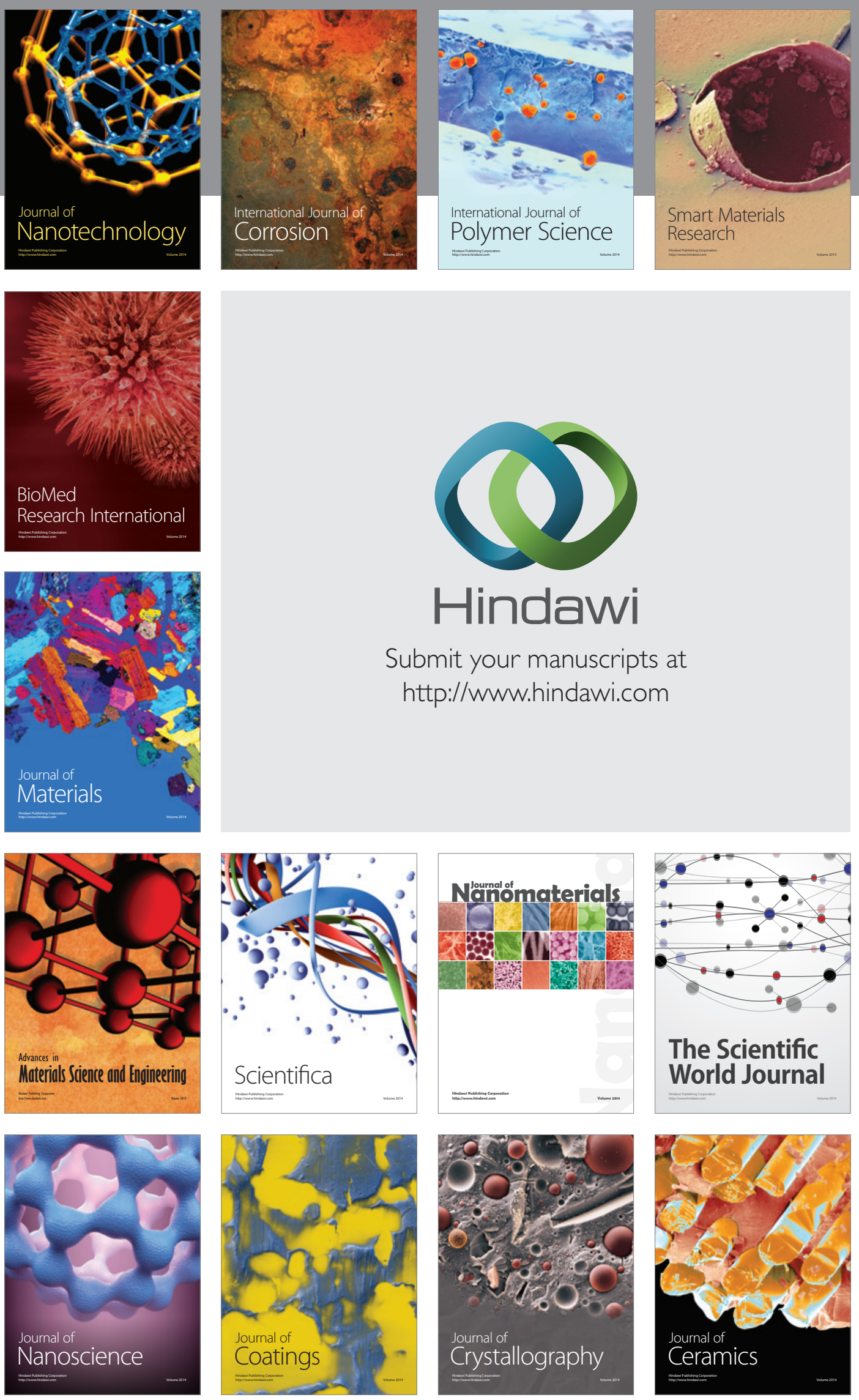

The Scientific World Journal

Submit your manuscripts at

http://www.hindawi.com

\section{World Journal}

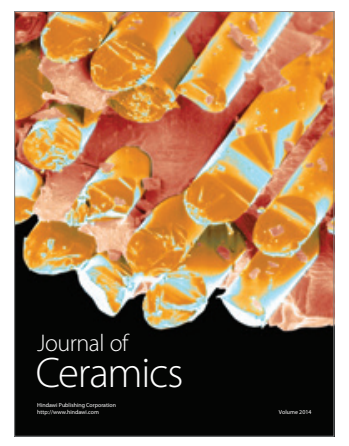

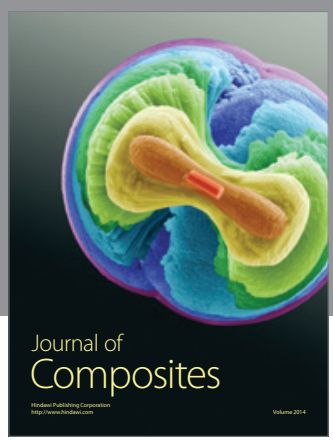
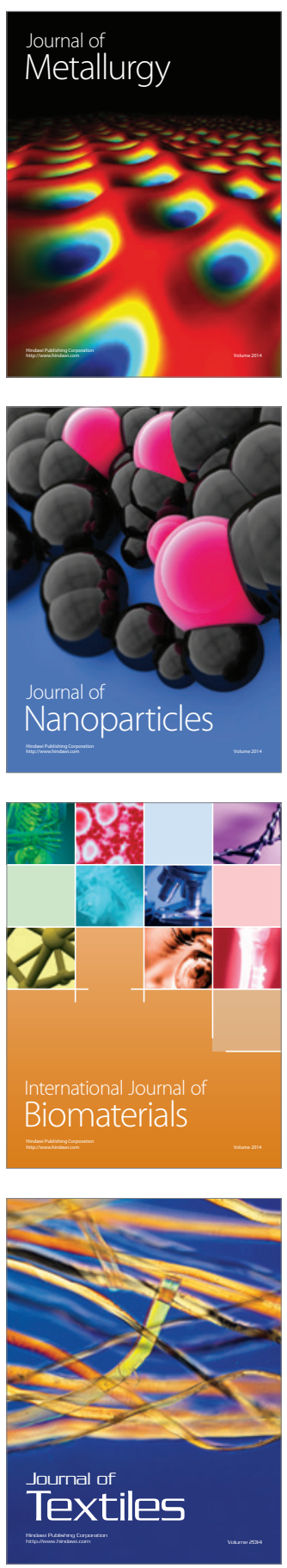